\title{
Limit theorems for discretely observed stochastic volatility models
}

\author{
VALENTINE GENON-CATALOT ${ }^{1}$, THIERRY JEANTHEAU ${ }^{1}$ and \\ CATHERINE LAREDO ${ }^{2 *}$
}

${ }^{1}$ Université de Marne-la-Vallée, Equipe d'Analyse et de Mathématiques Appliquées, 2 Rue de la Butte Verte, 93166 Noisy-Le-Grand Cédex, France

${ }^{2}$ Institut National de la Recherche Agronomique, Laboratoire de Biométrie, 78350 Jouy-en-Josas,

France

A general set-up is proposed to study stochastic volatility models. We consider here a two-dimensional diffusion process $\left(Y_{t}, V_{t}\right)$ and assume that only $\left(Y_{t}\right)$ is observed at $n$ discrete times with regular sampling interval $\Delta$. The unobserved coordinate $\left(V_{t}\right)$ is an ergodic diffusion which rules the diffusion coefficient (or volatility) of $\left(Y_{t}\right)$. The following asymptotic framework is used: the sampling interval tends to 0 , while the number of observations and the length of the observation time tend to infinity. We study the empirical distribution associated with the observed increments of $\left(Y_{t}\right)$. We prove that it converges in probability to a variance mixture of Gaussian laws and obtain a central limit theorem. Examples of models widely used in finance, and included in this framework, are given.

Keywords: diffusion processes; discrete time observations; empirical distributions; limit theorems; mathematical finance; stochastic volatility

\section{Introduction}

Diffusion processes are now widely used for modelling purposes in various fields and especially in finance. Many papers are devoted to the estimation of drift and diffusion coefficients of such models. The diffusion sample path may be continuously or discretely observed and numerous asymptotic results have been obtained. Among many references, let us quote Kutoyants (1984), Dacunha-Castelle and Florens-Zmirou (1986), Donahl (1987), Genon-Catalot (1990), Larédo (1990), Genon-Catalot and Jacod (1993), Bibby and Sorensen (1995) and Kessler (1995). In these studies, when multidimensional diffusions are considered, all coordinates are assumed to be observed.

However, the case of an unobserved or hidden coordinate may occur in practice. Examples can be found in the field of finance with the introduction of the stochastic volatility models. Indeed, in this area, the term volatility stands for the diffusion coefficient of stock returns. The seminal model of Black and Scholes (1973) assumes that stock price processes follow a geometric Brownian motion with constant volatility, but recent empirical evidence has extensively shown that this constant volatility assumption is unrealistic (see, for example,

\footnotetext{
*To whom correspondence should be addressed. e-mail: cl@baobab.jouy.inra.fr.
} 
Bollerslev et al. (1992) for a survey paper, and Taylor and Xu (1994, 1995) among many others). As an answer, some researchers have proposed models which include stochastic volatility in such a way that the couple (stock price, volatility) still behaves as a twodimensional diffusion process (Hull and White (1987); Wiggins (1987); Scott (1987); Chesney and Scott (1989); Stein and Stein (1991); Heston (1993); Leblanc (1996)). This raises a difficulty since only the stock price is observable, while the volatility is unobservable.

Indeed, assume that $\left(S_{t}\right)$ is the price process of some stock or asset and that the stock returns are ruled as usual by

$$
\frac{\mathrm{d} S_{t}}{S_{t}}=\mu_{t} \mathrm{~d} t+\sigma_{t} \mathrm{~d} B_{t}
$$

Here, $\left(\sigma_{t}\right)$ is the volatility of $\left(S_{t}\right)$, and $\left(B_{t}\right)$ is a Brownian motion. In the first models, $\mu_{t}$ and $\sigma_{t}$ are constants or deterministic functions of $t$. It follows from the discussion above that $V_{t}=\sigma_{t}^{2}$ can be modelled by a diffusion process, driven by another Brownian motion $\left(W_{t}, t \geqslant 0\right)$.

In this set-up, Nelson (1992) and Foster and Nelson (1994) give a solution to the problem of filtering $\left(V_{t}\right)$ through $\left(S_{t}\right)$, i.e., they define estimators $\hat{V}_{t}$ of $V_{t}$ given the data up to time $t$ of $\left(S_{u}\right)$. Our concern here is different. It is not to estimate the stochastic value $V_{t}$, but to get information on the distribution of the $\left(V_{t}\right)$ process. So we are in the presence of a hidden Markov process. Most references in this field are concerned with discrete-time models, e.g., hidden Markov chains (see, for example, Elliott et al. (1995)). However, among the references quoted above, a few continuous-time models have been studied; in these papers, some estimators of parameters present in the $\left(V_{t}\right)$ model are computed in specific cases, using stock prices data, with heuristic and empirical approach, but no theoretical study.

Our aim here is to propose a general set-up to study stochastic volatility models. Let us consider a two-dimensional diffusion $\left(Y_{t}, V_{t}\right)$ such that

$$
\begin{array}{cl}
\mathrm{d} Y_{t}=\varphi\left(V_{t}\right) \mathrm{d} t+V_{t}^{1 / 2} \mathrm{~d} B_{t}, & Y_{0}=0, \\
\mathrm{~d} V_{t}=b\left(V_{t}\right) \mathrm{d} t+a\left(V_{t}\right) \mathrm{d} W_{t}, & V_{0}=\eta,
\end{array}
$$

where $\left(B_{t}, W_{t}\right)_{t \geqslant 0}$ is a two-dimensional standard Brownian motion, $\left(V_{t}\right)$ is a positive diffusion process and $\eta$ is a positive random variable independent of $\left(B_{t}, W_{t}\right)_{t \geqslant 0}$.

In the model equations above, $Y_{t}$ stands for $\log S_{t}$. The sample path $\left(Y_{t}\right)$ is discretely observed at regularly spaced times $t_{i}=i \Delta, i=0, \ldots, n$. The aim is to get information on the $\left(V_{t}\right)$ model from the observations $\left(Y_{t_{1}}, \ldots, Y_{t_{n}}\right)$ only. We introduce here the appropriate assumptions and asymptotic framework. The key assumption is that the diffusion $\left(V_{t}\right)$ has to be ergodic. Apart from this assumption, the diffusion model for $\left(V_{t}\right)$ can be quite general; so our results will apply to most of the specific models proposed in the financial literature.

Our asymptotic framework is as follows. The number of observations $n$ tends to infinity, the sampling interval $\Delta=\Delta_{n}$ tends to zero, and the length of the observation time interval $n \Delta_{n}$ tends to infinity. We investigate the asymptotic behaviour of the empirical distribution $\hat{P}_{n}$ associated with the random variables

$$
X_{i}=X_{i}^{n}=\frac{1}{\Delta_{n}^{1 / 2}}\left(Y_{t_{i}}-Y_{t_{i-1}}\right),
$$


where $t_{i}=t_{i}^{n}=i \Delta_{n}, i=0, \ldots, n$, i.e.,

$$
\hat{P}_{n}=\frac{1}{n} \sum_{i=1}^{n} \delta_{X_{i}^{n}}
$$

where $\delta_{a}$ is the Dirac measure at point $a$.

The paper is organized as follows. In Sections 2 and 3, we set $\varphi=0$. We assume that $\left(V_{t}\right)$ is a strictly stationary positive process with stationary distribution $\pi(v) \mathrm{d} v$ on $(0,+\infty)$.

The main result of this paper is a convergence theorem for

$$
\hat{P}_{n}(f)=\int_{\mathbb{R}} f(x) \mathrm{d} \hat{P}_{n}(x)=\frac{1}{n} \sum_{i=1}^{n} f\left(X_{i}^{n}\right),
$$

where $f$ belongs to a class of functions $\mathscr{F}$ satisfying appropriate conditions. This class contains the functions $f(x)=x^{2 p}, p \geqslant 1$ such that $\mathrm{E} \eta^{2 p}$ is finite. We prove in Theorem 1 that $\hat{P}_{n}(f)$ converges in probability to $P_{\pi}(f)$, where $P_{\pi}$ is the distribution of $X=\epsilon \eta^{1 / 2}$ and $(\epsilon, \eta)$ has distribution $\mathscr{N}(0,1) \otimes \pi(v) \mathrm{d} v$. Therefore, the distribution $P_{\pi}$ is a variance mixture of Gaussian laws, the mixing distribution being the stationary distribution of the unobservable diffusion $\left(V_{t}\right)$.

In Theorem 2, we prove that, under the additional assumption $n \Delta_{n}^{2} \rightarrow 0$,

$$
\left(n \Delta_{n}\right)^{1 / 2}\left[\hat{P}_{n}(f)-P_{\pi}(f)\right]
$$

converges in distribution to a Gaussian distribution $\mathscr{N}(0, V(f))$. A difficulty arises when checking $V(f)<+\infty$. For this, we give a sufficient condition (Proposition 1) involving only the distribution $\pi$, which implies that $V(f)$ is finite for any $f$ in $\mathscr{F}$. The convergence in distribution also holds for finite-dimensional distributions (Theorem 3).

In Section 4 , we study the case $\varphi \neq 0$. Under appropriate assumptions on $\varphi$, the results of Theorems 1 and 2 are unchanged (Theorem 4). This implies that $\varphi$ may be known or unknown. Section 5 is devoted to the study of related models. We investigate the case where $V_{t}=\sigma_{t}^{2}$ with $\left(\sigma_{t}\right)$ a stationary diffusion on $\mathbb{R}$. This includes the case of $\left(\sigma_{t}\right)$ a Gaussian diffusion. Some examples are given in Section 6.

The results obtained here clearly have several statistical implications. The empirical distribution $\hat{P}_{n}$ can be used to estimate the stationary distribution $\pi$ of the unobserved diffusion $\left(V_{t}\right)$. This subject will be investigated in a forthcoming paper.

\section{Model and assumptions}

\subsection{The model}

Let $\left(Y_{t}, V_{t}\right)_{t \geqslant 0}$ be the two-dimensional diffusion process defined as the solution of

$$
\begin{array}{ll}
\mathrm{d} Y_{t}=V_{t}^{1 / 2} \mathrm{~d} B_{t}, & Y_{0}=0, \\
\mathrm{~d} V_{t}=b\left(V_{t}\right) \mathrm{d} t+a\left(V_{t}\right) \mathrm{d} W_{t}, & V_{0}=\eta,
\end{array}
$$


where

(1) $\left(B_{t}, W_{t}\right)_{t \geqslant 0}$ is a standard Brownian motion of $\mathbb{R}^{2}$ defined on a probability space $(\Omega, \mathscr{C}, \mathbf{P})$ and

(2) $\eta$ is a random variable defined on $\Omega$, independent of $\left(B_{t}, W_{t}\right)_{t \geqslant 0}$.

Equation (4) defines a one-dimensional diffusion process. We make now the classical assumptions on functions $b$ and $a$ which ensure that the solution of (4) is a positive recurrent diffusion on $(0,+\infty)$ and a strictly stationary and ergodic process.

(A1) $b, a$ are continuous real functions on $\mathbb{R}$, and $C^{1}$ functions on $(0,+\infty)$ such that

$$
\exists k>0, \quad \forall v>0, \quad b^{2}(v)+a^{2}(v) \leqslant k\left(1+v^{2}\right) \quad \text { and } \quad \forall v>0, \quad a(v)>0 .
$$

For $v_{0}>0$, define

$$
s(v)=\exp \left(-2 \int_{v_{0}}^{v} \frac{b(u)}{a^{2}(u)} \mathrm{d} u\right)
$$

(A2)

$$
\int_{0+} s(v) \mathrm{d} v=+\infty, \quad \int^{+\infty} s(v) \mathrm{d} v+\infty, \quad \int_{0}^{+\infty} \frac{\mathrm{d} v}{a^{2}(v) s(v)}=M<+\infty
$$

Let

$$
\pi(v)=\frac{1}{M} \frac{1}{a^{2}(v) s(v)} 1_{(v>0)}
$$

(A3) The initial random variable $\eta$ has distribution $\pi(v) \mathrm{d} v$.

(A4) $\exists \gamma \geqslant 2, \int_{0}^{+\infty} v^{\gamma} \pi(v) \mathrm{d} v<+\infty$.

Assumptions (A1) and (A2) ensure existence and uniqueness of the solution of (4) together with the positive recurrence on $(0,+\infty)$. In particular, the following holds:

$$
\mathbf{P}\left(V_{t}>0, \forall t \geqslant 0\right)=1 .
$$

Assumption (A3) provides the strict stationarity property. Note that, for each $t \geqslant 0, V_{t}$ has distribution $\pi(v) \mathrm{d} v$ which satisfies, under (A4), $\mathrm{E} V_{t}^{\gamma}<\infty$. So we introduce the notation

$$
m(p)=\int_{0}^{+\infty} v^{p} \pi(v) \mathrm{d} v=\mathrm{E} V_{t}^{p} \quad \text { for } 0 \leqslant p \leqslant \gamma .
$$

Comment 1. We are aware that, in the financial papers, more general models are considered. The two Brownian motions $\left(B_{t}\right)$ and $\left(W_{t}\right)$ are supposed to be correlated; the drift function of $\left(Y_{t}\right)$ may depend on $t, Y_{t}$ and $V_{t}$. Here, we have chosen to simplify the model in order to clarify the statistical problems involved in this class of models. 


\subsection{Observations and asymptotic framework}

The diffusion $\left(V_{t}\right)$ is supposed to be unobservable but it rules the conditional variance of $\left(Y_{t}\right)$ (see (3) and (4)). We assume that $\left(Y_{t}\right)$ is observable but only at $n$ discrete times with regular sampling interval $\Delta$. We denote by $t_{i}=i \Delta, i=0, \ldots, n$, these observation times. Our results are obtained in the asymptotic framework defined as follows. As $n \rightarrow+\infty$,

$$
\Delta=\Delta_{n} \rightarrow 0 \quad \text { and } \quad T_{n}=n \Delta_{n} \rightarrow+\infty .
$$

The length $T_{n}$ of the time interval where observations are available tends to infinity, while the sampling interval tends to 0 .

Comment 2. In order to justify the double asymptotic framework, let us point out that a discrete $\Delta$-sampling observation of $\left(Y_{t}\right)$ on a fixed time interval provides information on the conditional variance $V_{T}$ as $\Delta \rightarrow 0$. This is the filtering approach of Nelson (1992). To go further and obtain estimators for parameters in $b($.$) and a($.$) , we have to use the ergodicity$ properties of $\left(V_{t}\right)$, and so let $T=n \Delta_{n}$ tends to infinity. We refer for this to a forthcoming paper.

Our concern is the study of the asymptotic behaviour of $\hat{P}_{n}(f)$ (see (2)). In the next section, we introduce the appropriate class of functions $f$ for this study.

\subsection{The class of functions}

For $f: \mathbb{R} \rightarrow \mathbb{R}$, let us consider, when defined, the function $h_{f}: \mathbb{R}^{+} \rightarrow \mathbb{R}$ :

$$
h_{f}(v)=\int_{\mathbb{R}} f\left(x v^{1 / 2}\right) n(x) \mathrm{d} x,
$$

where $n(x)=1 /(2 \pi)^{1 / 2} \exp \left(-x^{2} / 2\right)$ is the $\mathscr{N}(0,1)$ density. We consider the following conditions.

(C1) Function $f$ is $C^{1}$ and such that, for the exponent $\gamma$ given in (A4),

$$
\exists K_{1}>0, \forall x \in \mathbb{R}, \quad|f(x)| \leqslant K_{1}\left(1+|x|^{\gamma}\right) .
$$

(C2) Function $f$ is such that $h_{f} \in C^{1}[0,+\infty)$ and, for $\gamma$ given in (A4),

$$
\exists K_{2}>0, \forall v \geqslant 0, \quad\left|h_{f}^{\prime}(v)\right| \leqslant K_{2}\left(1+v^{\gamma / 2}\right) .
$$

Denote by $\mathscr{F}_{\gamma}$ the class of functions satisfying $(\mathrm{C} 1)$ and $(\mathrm{C} 2)$. Under $(\mathrm{C} 1)$, the function $h_{f}$ is well defined and continuous and satisfies $h_{f}(v)=\mathrm{E} f\left(\epsilon v^{1 / 2}\right)$, where $\epsilon$ is a $\mathscr{N}(0,1)$ random variable. Let us point out that, for $f \in \mathscr{F}_{\gamma}$, using (C1), (C2) and (A4),

$$
\mathrm{E} h_{f}^{2}\left(V_{t}\right)=\mathrm{E} h_{f}^{2}(\eta)<+\infty, \quad \mathrm{E} h_{f}^{\prime 2}\left(V_{t}\right)=\mathrm{E} h_{f}^{\prime 2}(\eta)<+\infty .
$$

Note that $\mathrm{E} h_{f}(\eta)=\mathrm{E} f\left(\epsilon \eta^{1 / 2}\right)$ where $(\epsilon, \eta)$ has distribution $\mathscr{N}(0,1) \otimes \pi(v) \mathrm{d} v$. Therefore, the distribution of $\epsilon \eta^{1 / 2}$ is a variance mixture of Gaussian laws, the mixing distribution being the stationary distribution of $V_{t}$. We shall denote this distribution by $P_{\pi}$. For $f \in \mathscr{F}_{\gamma}$, we have 


$$
P_{\pi}(f)=\int_{\mathbb{R}} f(x) \mathrm{d} P_{\pi}(x)=\iint f\left(\epsilon v^{1 / 2}\right) n(x) \pi(v) \mathrm{d} x \mathrm{~d} v=\int_{\mathbb{R}^{+}} h_{f}(v) \pi(v) \mathrm{d} v .
$$

Let us give examples of functions $f$ belonging to $\mathscr{F}_{\gamma}$.

(1) For $t \in \mathbb{R}$ and $f(x)=\exp (\mathrm{i} t x), h_{f}(v)=\exp \left(-t^{2} v / 2\right)$, and $P_{\pi}(f)=\mathrm{E}\left[\exp \left(-t^{2} \eta / 2\right)\right]$. Although in this example, the function $f$ is complex valued, the results below will be true because $h_{f}$ is a real-valued function so that $P_{\pi}(f)$ is real.

(2) For $1 \leqslant p \leqslant \gamma / 2, f(x)=x^{2 p}, h_{f}(v)=C_{2} v^{p}$, where

$$
C_{k}=\int_{\mathbb{R}}|x|^{k} n(x) \mathrm{d} x
$$

is the $k$ th absolute moment of the distribution $\mathscr{N}(0,1)$ and, using (7) and (12),

$$
P_{\pi}(f)=C_{2 p} \mathrm{E} \eta^{p}=C_{2 p} m(p) .
$$

(3) This is a generalization of Example 2. Set $f(x)=g\left(x^{2}\right), g \in C^{1}[0,+\infty)$, and

$$
\exists K>0, \quad \forall u \geqslant 0, \quad|g(u)|+\left|g^{\prime}(u)\right| \leqslant K\left(1+u^{\gamma / 2}\right) .
$$

Finally, we introduce the function

$$
A_{f}(v)=\int_{0}^{v}\left[h_{f}(u)-P_{\pi}(f)\right] \pi(u) \mathrm{d} u .
$$

Note that $A_{f}(+\infty)=0$.

\section{Limit theorems for the empirical distribution}

\subsection{Main results}

We successively state the convergence in probability of $\hat{P}_{n}(f)$ and then the central limit theorem.

Theorem 1. Assume (A1)-(A4) and let $f$ belong to $\mathscr{F}_{\gamma}$. Then, if $\Delta_{n} \rightarrow 0$ and $n \Delta_{n} \rightarrow+\infty$,

$$
\hat{P}_{n}(f) \underset{n \rightarrow+\infty}{\rightarrow} P_{\pi}(f) \text { in probability. }
$$

Going a step further into the analysis, we define

$$
V(f)=4 M \int_{0}^{+\infty} s(v) A_{f}^{2}(v) \mathrm{d} v,
$$

where $s($.$) and M$ are defined in (A2) and $A_{f}(v)$ is given in (14). Let us notice that the formula for $V(f)$ does not depend on the choice of the value $v_{0}$ used to compute $s(v)$ (see assumption (A2)). This is because the product $M s(v)$ does not depend on $v_{0}$. 
Theorem 2. Assume (A1)-(A4) and let $f$ belong to $\mathscr{F}_{\gamma}$. Assume that $\Delta_{n} \rightarrow 0$ and $n \Delta_{n} \rightarrow+\infty$. If, moreover, $n \Delta_{n}^{2} \rightarrow 0$ and $V(f)<+\infty$, then

$$
\left(n \Delta_{n}\right)^{1 / 2}\left[\hat{P}_{n}(f)-P_{\pi}(f)\right] \underset{n \rightarrow+\infty}{\stackrel{\mathscr{D}}{\rightarrow}} \mathscr{N}(0, V(f)) .
$$

If $f(x)=x^{2}$, the additional condition $n \Delta_{n}^{2} \rightarrow 0$ is not needed.

Proofs of both theorems rely upon lemmas that we state now.

\subsection{Preliminary lemmas}

Let us define

$$
\bar{V}_{i}=\frac{1}{\Delta_{n}} \int_{t_{i-1}}^{t_{i}} V_{s} \mathrm{~d} s
$$

Lemma 1. Assume (A1)-(A4). Then, for $1 \leqslant p \leqslant \gamma$,

$$
\mathrm{E} \bar{V}_{i}^{p} \leqslant m(p)
$$

where $m(p)$ is given in (7).

Proof. Since $p \geqslant 1$, the Hölder inequality gives

$$
\bar{V}_{i}^{p} \leqslant \frac{1}{\Delta_{n}} \int_{t_{i-1}}^{t_{i}} V_{s}^{p} \mathrm{~d} s
$$

which, by stationarity, gives the result.

Lemma 2. Assume (A1)-(A4). There exists a constant $C$ such that, for $0 \leqslant v \leqslant 1$,

$$
\mathrm{E}\left(V_{v}-V_{0}\right)^{2} \leqslant C v
$$

where $C$ only depends on $m(2)$ and $k$ (see (A1) and (7)).

Proof. This lemma is a straightforward consequence of our assumptions. We have

$$
V_{v}-V_{0}=\int_{0}^{v} b\left(V_{u}\right) \mathrm{d} u+\int_{0}^{v} a\left(V_{u}\right) \mathrm{d} W_{u} .
$$

Thus, it follows that

$$
\left(V_{v}-V_{0}\right)^{2} \leqslant 2\left(\int_{0}^{v} b\left(V_{u}\right) \mathrm{d} u\right)^{2}+2\left(\int_{0}^{v} a\left(V_{u}\right) \mathrm{d} W_{u}\right)^{2} \leqslant 2 v \int_{0}^{v} b^{2}\left(V_{u}\right) \mathrm{d} u+2\left(\int_{0}^{v} a\left(V_{u}\right) \mathrm{d} W_{u}\right)^{2}
$$


By (A1), we obtain

$$
\mathrm{E}\left(V_{v}-V_{0}\right)^{2} \leqslant 2 k\left(v \int_{0}^{v}\left[1+\mathrm{E}\left(V_{u}^{2}\right)\right] \mathrm{d} u+\int_{0}^{v}\left[1+\mathrm{E}\left(V_{u}^{2}\right)\right] \mathrm{d} u\right) .
$$

Now, by $\quad(\mathrm{A} 4), \quad \mathrm{E} V_{u}^{2}=\int v^{2} \pi(v) \mathrm{d} v=m(2)<+\infty . \quad$ Hence, $\quad \mathrm{E}\left(V_{v}-V_{0}\right)^{2} \leqslant 2 k[1+$ $m(2)]\left(v^{2}+v\right)$. This gives the lemma.

Lemma 3. Assume (A1)-(A4). Let $\psi \in C^{1}[0,+\infty)$ be such that

$$
\exists K>0, \quad \forall v \geqslant 0, \quad\left|\psi^{\prime}(v)\right| \leqslant K\left(1+v^{\gamma / 2}\right) .
$$

Then,

$$
B_{n}(\psi)=\frac{1}{n} \sum_{i=1}^{n} \psi\left(\bar{V}_{i}\right)-\frac{1}{n \Delta_{n}} \int_{0}^{n \Delta_{n}} \psi\left(V_{s}\right) \mathrm{d} s=\Delta_{n}^{1 / 2} O_{P}(1) .
$$

Proof. First note that, if $\psi$ is linear, $B_{n}(\psi) \equiv 0$. Otherwise,

$$
B_{n}(\psi)=\frac{1}{n \Delta_{n}} \sum_{i=1}^{n} \int_{t_{i-1}}^{t_{i}}\left[\psi\left(\bar{V}_{i}\right)-\psi\left(V_{s}\right)\right] \mathrm{d} s .
$$

Applying the Taylor formula yields

$$
\psi\left(\bar{V}_{i}\right)-\psi\left(V_{s}\right)=\left(\bar{V}_{i}-V_{s}\right) R_{i}(s),
$$

with

$$
R_{i}(s)=\int_{0}^{1} \psi^{\prime}\left[V_{s}+u\left(\bar{V}_{i}-V_{s}\right)\right] \mathrm{d} u
$$

Now consider

$$
\bar{V}_{i}-V_{s}=\frac{1}{\Delta_{n}} \int_{t_{i-1}}^{t_{i}}\left(V_{t}-V_{s}\right) \mathrm{d} t
$$

Thus

$$
\left|B_{n}(\psi)\right| \leqslant \frac{1}{n \Delta_{n}^{2}} \sum_{i=1}^{n} \int_{t_{i-1}}^{t_{i}} \mathrm{~d} s \quad\left|R_{i}(s)\right| \int_{t_{i-1}}^{t_{i}}\left|V_{t}-V_{s}\right| \mathrm{d} t .
$$

Therefore, taking the expectation and then applying the Schwarz inequality lead to

$$
\mathrm{E}\left|B_{n}(\psi)\right| \leqslant \frac{1}{n \Delta_{n}^{2}} \sum_{i=1}^{n} \int_{t_{i-1}}^{t_{i}} \int_{t_{i-1}}^{t_{i}} \mathrm{~d} s \mathrm{~d} t\left[\mathrm{E}\left(V_{t}-V_{s}\right)^{2} \mathrm{E} R_{i}(s)^{2}\right]^{1 / 2} .
$$

Lemma 2 and the strict stationarity give, for $s, t \in\left[t_{i-1}, t_{i}\right]$ and $n$ large enough,

$$
\mathrm{E}\left(V_{t}-V_{s}\right)^{2}=\mathrm{E}\left(V_{|t-s|}-V_{0}\right)^{2} \leqslant C|t-s| .
$$

Coming back to $R_{i}(s)$, we have, using the assumption on $\psi^{\prime}$, 


$$
\begin{gathered}
\left|R_{i}(s)\right| \leqslant K \int_{0}^{1}\left[1+\left|V_{s}+u\left(\bar{V}_{i}-V_{s}\right)\right|^{\gamma / 2}\right] \mathrm{d} u, \\
V_{s}+u\left(\bar{V}_{i}-V_{s}\right)=\frac{1}{\Delta_{n}} \int_{t_{i-1}}^{t_{i}}\left[u V_{t}+(1-u) V_{s}\right] \mathrm{d} t .
\end{gathered}
$$

Thus, by Hölder's inequality, since $\gamma / 2 \geqslant 1$,

$$
\left|V_{s}+u\left(\bar{V}_{i}-V_{s}\right)\right|^{\gamma / 2} \leqslant \frac{1}{\Delta_{n}} \int_{t_{i-1}}^{t_{i}}\left|u V_{t}+(1-u) V_{s}\right|^{\gamma / 2} \mathrm{~d} t \leqslant \frac{K(\gamma)}{\Delta_{n}} \int_{t_{i-1}}^{t_{i}}\left(V_{t}^{\gamma / 2}+V_{s}^{\gamma / 2}\right) \mathrm{d} t
$$

for some constant $K(\gamma)$ and for all $u \in[0,1]$. So,

$$
\left|R_{i}(s)\right| \leqslant K K(\gamma)\left(1+\frac{1}{\Delta_{n}} \int_{t_{i-1}}^{t_{i}}\left(V_{t}^{\gamma / 2}+V_{s}^{\gamma / 2}\right) \mathrm{d} t\right)
$$

and

$$
R_{i}(s)^{2} \leqslant L\left(1+\frac{1}{\Delta_{n}} \int_{t_{i-1}}^{t_{i}}\left(V_{t}^{\gamma}+V_{s}^{\gamma}\right) \mathrm{d} t\right)
$$

for some constant $L>0$. Using now assumption (A4), we obtain

$$
\mathrm{E} R_{i}(s)^{2} \leqslant L[1+2 m(\gamma)]
$$

Applying inequalities (18) and (19) to (17) leads to

$$
\mathrm{E}\left|B_{n}(\psi)\right| \leqslant\{C L[1+2 m(\gamma)]\}^{1 / 2} \frac{1}{n \Delta_{n}^{2}} \sum_{i=1}^{n} \int_{t_{i-1}}^{t_{i}} \int_{t_{i-1}}^{t_{i}} \mathrm{~d} s \mathrm{~d} t|t-s|^{1 / 2}
$$

By a straightforward computation

$$
\int_{t_{i-1}}^{t_{i}} \int_{t_{i-1}}^{t_{i}} \mathrm{~d} s \mathrm{~d} t|t-s|^{1 / 2}=\frac{8}{15} \Delta_{n}^{2} \Delta_{n}^{1 / 2}
$$

This implies that $\mathrm{E}\left|B_{n}(\psi)\right| \leqslant L^{\prime} \Delta_{n}^{1 / 2}$ for some constant $L^{\prime}>0$. Thus, we get the result of Lemma 3.

\subsection{Proofs of the theorems}

Let us now prove the theorems.

Proof of Theorem 1. Let us study $\hat{P}_{n}(f)-P_{\pi}(f)$ when $f$ belongs to $\mathscr{F}_{\gamma}$. Let $\mathscr{G}=$ $\sigma\left(V_{0}, W_{t}, t \geqslant 0\right)$, and denote by $\mathrm{E}^{\mathscr{G}}$ the conditional expectation with respect to $\mathscr{G}$. Conditionally on $\mathscr{F}$, the random variables $\left(X_{i}, i=1, \ldots, n\right)$ defined in (1) are independent and $X_{i}$ has distribution $\mathscr{N}\left(0, \bar{V}_{i}\right)$ where $\bar{V}_{i}$ is defined in (16). Moreover, let us check that 


$$
\mathrm{E}\left|f\left(X_{i}\right)\right|<\infty, \quad \mathrm{E}^{\mathscr{G}} f\left(X_{i}\right)=h_{f}\left(\bar{V}_{i}\right) .
$$

By (C1), $\left|f\left(X_{i}\right)\right| \leqslant K_{1}\left(1+\left|X_{i}\right|^{\gamma}\right)$. So, using Lemma 1, and (13) for the definition of $C_{\gamma}$,

$$
\mathrm{E}\left|X_{i}\right|^{\gamma}=\mathrm{EE}^{\mathscr{G}}\left|X_{i}\right|^{\gamma}=C_{\gamma} \mathrm{E} \bar{V}_{i}^{\gamma / 2} \leqslant C_{\gamma} m\left(\frac{\gamma}{2}\right)
$$

Thus, $\left|f\left(X_{i}\right)\right|$ has finite expectation and

$$
\mathrm{E}^{\mathscr{G}} f\left(X_{i}\right)=\int_{\mathbb{R}} f(x) \exp \left(-\frac{x^{2}}{2 \bar{V}_{i}}\right) \frac{\mathrm{d} x}{\left(2 \pi \bar{V}_{i}\right)^{1 / 2}}=\int_{\mathbb{R}} f\left(x \bar{V}_{i}^{1 / 2}\right) n(x) \mathrm{d} x=h_{f}\left(\bar{V}_{i}\right) .
$$

Now, we can split the difference $\hat{P}_{n}(f)-P_{\pi}(f)$ into the sum of three terms:

$$
\hat{P}_{n}(f)-P_{\pi}(f)=A_{n}+B_{n}+D_{n},
$$

with, using the notation introduced in Lemma 3,

$$
\begin{gathered}
A_{n}=\frac{1}{n} \sum_{i=1}^{n}\left[f\left(X_{i}\right)-\mathrm{E}^{\mathscr{G}} f\left(X_{i}\right)\right], \\
B_{n}=\frac{1}{n} \sum_{i=1}^{n} \mathrm{E}^{\mathscr{G}} f\left(X_{i}\right)-\frac{1}{n \Delta_{n}} \int_{0}^{n \Delta_{n}} h_{f}\left(V_{s}\right) \mathrm{d} s=B_{n}\left(h_{f}\right), \\
D_{n}=\frac{1}{n \Delta_{n}} \int_{0}^{n \Delta_{n}} h_{f}\left(V_{s}\right) \mathrm{d} s-\int_{0}^{\infty} h_{f}(v) \pi(v) \mathrm{d} v .
\end{gathered}
$$

Recall that

$$
P_{\pi}(f)=\int_{0}^{\infty} h_{f}(v) \pi(v) \mathrm{d} v
$$

Since $f$ satisfies (C2), we can apply Lemma 3 with $\psi=h_{f}$. Thus,

$$
B_{n}\left(h_{f}\right)=\Delta_{n}^{1 / 2} O_{P}(1) .
$$

We may apply to $\left(V_{t}\right)$ an ergodic theorem for one-dimensional positive recurrent diffusion (see, for example, Rogers and Williams (1987, p. 300)). Since $n \Delta_{n} \rightarrow+\infty$, we have, almost surely,

$$
\frac{1}{n \Delta_{n}} \int_{0}^{n \Delta_{n}} h_{f}\left(V_{s}\right) \mathrm{d} s \underset{n \rightarrow+\infty}{\rightarrow} \int_{0}^{\infty} h_{f}(v) \pi(v) \mathrm{d} v
$$

Thus,

$$
D_{n} \underset{n \rightarrow+\infty}{\rightarrow} 0 \text { almost surely. }
$$


Let us now study $A_{n}$. For this, set

$$
\chi_{i}^{n}=\frac{1}{n}\left[f\left(X_{i}\right)-\mathrm{E}^{\mathscr{G}} f\left(X_{i}\right)\right]
$$

Conditionally on $\mathscr{G}$, the random variables $\chi_{i}^{n}$ are centred and independent. Thus,

$$
\mathrm{E}^{\mathscr{G}}\left(A_{n}^{2}\right)=\sum_{i=1}^{n} \mathrm{E}^{\mathscr{G}}\left(\chi_{i}^{n}\right)^{2}
$$

Now

$$
\mathrm{E}^{\mathscr{G}}\left(\chi_{i}^{n}\right)^{2}=\frac{1}{n^{2}}\left\{\mathrm{E}^{\mathscr{G}} f^{2}\left(X_{i}\right)-\left[\mathrm{E}^{\mathscr{G}} f\left(X_{i}\right)\right]^{2}\right\} \leqslant \frac{1}{n^{2}} \mathrm{E}^{\mathscr{G}} f^{2}\left(X_{i}\right)=\frac{1}{n^{2}} \int f^{2}\left(x \bar{V}_{i}^{1 / 2}\right) n(x) \mathrm{d} x .
$$

Using condition $(\mathrm{C} 1)$ leads to $f^{2}(x) \leqslant K_{1}^{\prime}\left(1+x^{2 \gamma}\right)$. Thus,

$$
\mathrm{E}^{\mathscr{G}} f^{2}\left(X_{i}\right) \leqslant K_{1}^{\prime}\left(1+C_{2 \gamma} \bar{V}_{i}^{\gamma}\right)
$$

Therefore, by Lemma 1,

$$
\mathrm{E}\left(\chi_{i}^{n}\right)^{2} \leqslant \frac{1}{n^{2}} K_{1}^{\prime}\left[1+C_{2 \gamma} m(\gamma)\right]
$$

This implies that

$$
\mathrm{E} A_{n}^{2} \leqslant \frac{1}{n} K_{1}^{\prime}\left[1+C_{2 \gamma} m(\gamma)\right]
$$

Thus,

$$
A_{n}=n^{-1 / 2} O_{\mathbf{P}}(1)
$$

Joining (24)-(26) achieves the proof of Theorem 1.

Proof of Theorem 2. We study now $\left(n \Delta_{n}\right)^{1 / 2}\left[\hat{P}_{n}(f)-P_{\pi}(f)\right]$. Using the notation in (21)(23),

$$
\left(n \Delta_{n}\right)^{1 / 2}\left[\hat{P}_{n}(f)-P_{\pi}(f)\right]=\left(n \Delta_{n}\right)^{1 / 2} A_{n}+\left(n \Delta_{n}\right)^{1 / 2} B_{n}\left(h_{f}\right)+\left(n \Delta_{n}\right)^{1 / 2} D_{n}
$$

Two cases may occur; if $f(x)=x^{2}$, the function $h_{f}(v) \equiv v$, and, in this case, $B_{n}\left(h_{f}\right) \equiv 0$. Otherwise, it follows from (24) that

$$
\left(n \Delta_{n}\right)^{1 / 2} B_{n}\left(h_{f}\right)=\left(n \Delta_{n}^{2}\right)^{1 / 2} O_{\mathbf{P}}(1) .
$$

Hence, if $n \Delta_{n}^{2} \rightarrow 0$ as $n \rightarrow+\infty$, this term will asymptotically vanish. From (26), we see that 


$$
\left(n \Delta_{n}\right)^{1 / 2} A_{n}=\Delta_{n}^{1 / 2} O_{\mathbf{P}}(1)
$$

which also goes to 0 .

We only have to study the convergence in distribution of the third term of (27). It is equal to

$$
\left(n \Delta_{n}\right)^{1 / 2} D_{n}=\frac{1}{\left(n \Delta_{n}\right)^{1 / 2}} \int_{0}^{n \Delta_{n}}\left(h_{f}\left(V_{s}\right)-\int_{0}^{\infty} h_{f}(v) \pi(v) \mathrm{d} v\right) \mathrm{d} s .
$$

In order to apply a central limit theorem for one-dimensional positive recurrent diffusions (see, for example, Florens-Zmirou (1984)), let us set

$$
H_{f}(u)=h_{f}(u)-\int_{0}^{\infty} h_{f}(v) \pi(v) \mathrm{d} v .
$$

Clearly, the following holds:

$$
\int_{0}^{\infty} H_{f}(v) \pi(v) \mathrm{d} v=0
$$

Therefore,

$$
\frac{1}{\left(n \Delta_{n}\right)^{1 / 2}} \int_{0}^{n \Delta_{n}} H_{f}\left(V_{s}\right) \mathrm{d} s \underset{n \rightarrow+\infty}{\stackrel{\mathscr{D}}{\rightarrow}} \mathscr{N}(0, V(f)),
$$

where $V(f)$ is given by the formula

$$
V(f)=4 \int_{0}^{\infty} \pi(v)\left(s(v) a(v) \int_{0}^{v} \mathrm{~d} u \frac{H_{f}(u)}{a^{2}(u) s(u)}\right)^{2} \mathrm{~d} v .
$$

Definitions (5), (6), (9) and (14) lead to the expression of $V(f)$ given in (15).

Consequence. Let us consider sampling intervals $\Delta_{n}=n^{-\alpha}$. The conditions $\Delta_{n} \rightarrow 0$ and $n \Delta_{n} \rightarrow+\infty$ lead to $0<\alpha<1$. The additional condition $n \Delta_{n}^{2} \rightarrow 0$ leads to $\frac{1}{2}<\alpha<1$. Therefore, the rate of convergence of Theorem 2 is $\left(n \Delta_{n}\right)^{1 / 2}=n^{(1-\alpha) / 2}$ with $0<$ $(1-\alpha) / 2<\frac{1}{4}$.

\subsection{Some bounds for the asymptotic variance}

The result of Theorem 2 holds under the assumption that the quantity $V(f)$ defined in (15) is finite. For this, we have to study the behaviour around 0 and $+\infty$ of the function $s(v) A_{f}^{2}(v)$. The proposition below provides explicit bounds which can be easily computed.

Proposition 1. Assume (A1)-(A4) and

(i) $\int_{0^{+}} \mathrm{d} v s(v)\left(\int_{0}^{v} \pi(u) \mathrm{d} u\right)^{2}<+\infty$,

and 
(ii) $\int^{\infty} \mathrm{d} v s(v)\left(\int_{v}^{+\infty} u^{\gamma / 2} \pi(u) \mathrm{d} u\right)^{2}<+\infty$,

then, for all $f \in \mathscr{F}_{\gamma}, V(f)<+\infty$.

Proof. This result is a straightforward consequence of the following lemma.

Lemma 4. Assume (A1)-(A4) and let $f$ belong to $\mathscr{F}_{\gamma}$. Then,

(i) there exist $C>0$ and $v_{0}>0$ such that

$$
\forall v \in\left[0, v_{0}\right], \quad\left|A_{f}(v)\right| \leqslant C \int_{0}^{v} \pi(u) \mathrm{d} u
$$

and

(ii) there exist $C^{\prime}>0$ and $v_{1}>0$ such that

$$
\forall v \geqslant v_{1}, \quad\left|A_{f}(v)\right| \leqslant C^{\prime} \int_{v}^{+\infty} u^{\gamma / 2} \pi(u) \mathrm{d} u .
$$

Proof. Condition (C1) states that

$$
\exists K_{1}>0, \quad \forall x \in \mathbb{R}, \quad|f(x)| \leqslant K_{1}\left(1+|x|^{\gamma}\right) .
$$

Therefore, see (9) and (13),

$$
\forall v \geqslant 0, \quad\left|h_{f}(v)\right| \leqslant K_{1}\left(1+C_{\gamma} v^{\gamma / 2}\right) .
$$

Now,

$$
\left|A_{f}(v)\right| \leqslant \int_{0}^{v}\left[\left|h_{f}(u)\right|+\left|P_{\pi}(f)\right|\right] \pi(u) \mathrm{d} u .
$$

This leads to (i).

To deal with the neighbourhood of $+\infty$, we first recall that $A_{f}(+\infty)=0$. Hence

$$
A_{f}(v)=-\int_{v}^{+\infty} \pi(u) \mathrm{d} u\left[h_{f}(u)-P_{\pi}(f)\right] .
$$

Therefore,

$$
\left|A_{f}(v)\right| \leqslant \int_{v}^{+\infty} \pi(u) \mathrm{d} u\left[\left|h_{f}(u)\right|+\left|P_{\pi}(f)\right|\right],
$$

which leads to (ii).

Remark. In some cases, $V(f)$ can be explicitly computed. However, Proposition 1 gives sufficient conditions for checking that $V(f)$ is finite. Both conditions are easy to check and interesting because they only involve the diffusion model for $\left(V_{t}\right)$ and the moment condition on the stationary distribution $\pi$. 


\subsection{Finite-dimensional distributions}

Theorem 2 can be extended to finite-dimensional distributions. Let $f, g$ be two functions in $\mathscr{F}_{\gamma}$. Set $V(f)=V(f, f)$ and

$$
V(f, g)=4 M \int_{0}^{\infty} s(v) A_{f}(v) A_{g}(v) \mathrm{d} v
$$

Theorem 3. Assume (A1)-(A4) and let $\left(f_{1}, \ldots, f_{k}\right)$ be a finite set of functions in $\mathscr{F}_{\gamma}$. Assume that $\Delta_{n} \rightarrow 0$ and $n \Delta_{n} \rightarrow+\infty$. If, moreover, $n \Delta_{n}^{2} \rightarrow 0$ and $V\left(f_{i}, f_{i}\right)<+\infty$ for $i=1, \ldots, k$, then the vector

$$
\left(n \Delta_{n}\right)^{1 / 2}\left[\hat{P}_{n}\left(f_{i}\right)-P_{\pi}\left(f_{i}\right)\right]_{1 \leqslant i \leqslant k}
$$

converges in distribution to $\mathscr{N}_{k}\left(0,\left(V\left(f_{i}, f_{j}\right)\right)_{1 \leqslant i, j \leqslant k}\right)$.

Proof. Clearly, the assumption $V\left(f_{i}, f_{i}\right)<\infty$ for $i=1, \ldots, k$ implies that all the quantities $V\left(f_{i}, f_{j}\right)$ are well defined. Now, let $\alpha_{1}, \ldots, \alpha_{k}$ be given real numbers, and set $f=\alpha_{1} f_{1}+$ $\ldots+\alpha_{k} f_{k}$. Then,

$$
\hat{P}_{n}(f)-P_{\pi}(f)=\sum_{i=1}^{k} \alpha_{i}\left[\hat{P}_{n}\left(f_{i}\right)-P_{\pi}\left(f_{i}\right)\right] .
$$

Since $h_{f}(v)=\sum_{i=1}^{k} \alpha_{i} h_{f_{i}}(v)$, the function $A_{f}(v)$ associated with $f$ satisfies

$$
A_{f}(v)=\sum_{i=1}^{k} \alpha_{i} A_{f_{i}}(v)
$$

and $V(f, f)$ has the expansion

$$
V(f, f)=\sum_{i=1}^{k} \alpha_{i}^{2} V\left(f_{i}, f_{i}\right)+2 \sum_{1 \leqslant i<j \leqslant k} \alpha_{i} \alpha_{j} V\left(f_{i}, f_{j}\right) .
$$

This gives the result.

\section{Extension to the presence of a drift in the $\left(Y_{t}\right)$ model}

Stochastic models for stock prices and instantaneous standard deviations of the stock returns are generally given by

$$
\begin{aligned}
\mathrm{d} S_{t} & =\mu S_{t} \mathrm{~d} t+V_{t}^{1 / 2} S_{t} \mathrm{~d} B_{t}, \\
\mathrm{~d} V_{t} & =b\left(V_{t}\right) \mathrm{d} t+a\left(V_{t}\right) \mathrm{d} W_{t} .
\end{aligned}
$$

Only $\left(S_{t}\right)$ is observed and, in the simplest case, $\mu$ is a deterministic constant. In order to recover the previous setting, let $Y_{t}=\log S_{t}$. Then, a standard application of the Ito formula yields 


$$
\mathrm{d} Y_{t}=\left(\mu-\frac{1}{2} V_{t}\right) \mathrm{d} t+V_{t}^{1 / 2} \mathrm{~d} B_{t}
$$

This example shows the interest of studying models where there is a drift term in $\left(Y_{t}\right)$ depending only on the instantaneous conditional variance $V_{t}$.

Let us now consider the model

$$
\begin{array}{ll}
\mathrm{d} Y_{t}=\varphi\left(V_{t}\right) \mathrm{d} t+V_{t}^{1 / 2} \mathrm{~d} B_{t}, & Y_{0}=0, \\
\mathrm{~d} V_{t}=b\left(V_{t}\right) \mathrm{d} t+a\left(V_{t}\right) \mathrm{d} W_{t} & V_{0}=\eta,
\end{array}
$$

with $\eta$ independent of $\left(B_{t}, W_{t}\right)_{t \geqslant 0}$. From now on, we assume (A1)-(A4). As previously, define

$$
X_{i}=\frac{1}{\Delta_{n}^{1 / 2}}\left(Y_{t_{i}}-Y_{t_{i-1}}\right), \quad i=1, \ldots, n,
$$

and introduce the random variables

$$
\xi_{i}=\frac{1}{\Delta_{n}^{1 / 2}} \int_{t_{i-1}}^{t_{i}} V_{s}^{1 / 2} \mathrm{~d} B_{s}, \quad i=1, \ldots, n .
$$

Clearly, the results of Section 3 apply to the $\xi_{i}$ variables. We obtain below that these results are still true when $X_{i}$ is substituted for $\xi_{i}$.

Let us define

$$
\hat{P}_{n}=\frac{1}{n} \sum_{i=1}^{n} \delta_{X_{i}}, \quad \hat{Q}_{n}=\frac{1}{n} \sum_{i=1}^{n} \delta_{\xi_{i}}
$$

Theorem 4. Assume (A1)-(A4). Let $f$ belong to $\mathscr{F}_{\gamma}$ and assume moreover that $f^{\prime}$ is such that

$$
\exists p, \quad 1 \leqslant p \leqslant \gamma, \quad \exists C, \quad \forall x \in \mathbb{R}, \quad\left|f^{\prime}(x)\right| \leqslant C\left(1+|x|^{p}\right) .
$$

Then, if the drift function $\varphi$ satisfies $\mathrm{E}|\varphi(\eta)|^{p+1}<+\infty$, one has

(i) if $\Delta_{n}$ tends to 0 and $n \Delta_{n}$ tends to infinity, Theorem 1 holds for $\hat{P}_{n}(f)$, and

(ii) if moreover $n \Delta_{n}^{2}$ tends to 0 , Theorem 2 holds for $\hat{P}_{n}(f)$.

Proof. Theorem 1 and Theorem 2 hold for $\hat{Q}_{n}$. So, only the difference $\hat{P}_{n}(f)-\hat{Q}_{n}(f)$ has to be studied. By the Taylor formula,

$$
\hat{P}_{n}(f)-\hat{Q}_{n}(f)=\frac{1}{n} \sum_{i=1}^{n}\left(X_{i}-\xi_{i}\right) \int_{0}^{1} f^{\prime}\left[\xi_{i}+u\left(X_{i}-\xi_{i}\right)\right] \mathrm{d} u .
$$

Note that $X_{i}-\xi_{i}=\Delta_{n}^{-1 / 2} \int_{t_{i-1}}^{t_{i}} \varphi\left(V_{s}\right) \mathrm{d} s$ are $\mathscr{G}$-measurable random variables where $\mathscr{G}=\sigma\left(V_{0},\left(W_{t}, t \geqslant 0\right)\right)$. Using the assumption on $f^{\prime}$,

$$
\left|f^{\prime}\left[\xi_{i}+u\left(X_{i}-\xi_{i}\right)\right]\right| \leqslant C\left(1+\left|\xi_{i}+u\left(X_{i}-\xi_{i}\right)\right|^{p}\right) \leqslant C^{\prime}\left(1+\left|\xi_{i}\right|^{p}+\left|X_{i}-\xi_{i}\right|^{p}\right),
$$

for all $u \in[0,1]$. Taking the conditional expectation with respect to $\mathscr{G}$ and using that $\xi_{i}$ is $\mathscr{N}\left(0, \bar{V}_{i}\right)$ lead to 


$$
\mathrm{E}^{\mathscr{G}}\left|f^{\prime}\left[\xi_{i}+u\left(X_{i}-\xi_{i}\right)\right]\right| \leqslant C^{\prime}\left(1+C_{p} \bar{V}_{i}^{p / 2}+\left|X_{i}-\xi_{i}\right|^{p}\right) .
$$

Recall that $\bar{V}_{i}=\left(1 / \Delta_{n}\right) \int_{t_{i-1}}^{t_{i}} V_{s} \mathrm{~d} s$ and that $C_{p}$ is the $p$ th absolute moment of the $\mathscr{N}(0,1)$ distribution. Therefore,

$$
\mathrm{E}\left|\hat{P}_{n}(f)-\hat{Q}_{n}(f)\right| \leqslant \frac{C^{\prime}}{n} \sum_{i=1}^{n} L_{i}
$$

with

$$
L_{i}=\mathrm{E}\left[\left|X_{i}-\xi_{i}\right|\left(1+C_{p} \bar{V}_{i}^{p / 2}+\left|X_{i}-\xi_{i}\right|^{p}\right)\right]
$$

For $k \geqslant 1$, one has

$$
\mathrm{E}\left|X_{i}-\xi_{i}\right|^{k}=\Delta_{n}^{k / 2} \mathrm{E}\left(\left|\frac{1}{\Delta n} \int_{t_{i-1}}^{t_{i}} \varphi\left(V_{s}\right) \mathrm{d} s\right|^{k}\right) \leqslant \Delta_{n}^{k / 2} \mathrm{E}|\varphi(\eta)|^{k} .
$$

Using now the Cauchy-Schwarz inequality, and applying Lemma 1 to $\bar{V}_{i}$, we get

$$
\mathrm{E}\left(\left|X_{i}-\xi_{i}\right| \bar{V}_{i}^{p / 2}\right) \leqslant\left[\mathrm{E}\left(X_{i}-\xi_{i}\right)^{2} \mathrm{E} \bar{V}_{i}^{p}\right]^{1 / 2} \leqslant \Delta_{n}^{1 / 2}\left[\mathrm{E} \varphi^{2}(\eta)\right]^{1 / 2} m(p)^{1 / 2} .
$$

Joining these results, we obtain

$$
L_{i} \leqslant \Delta_{n}^{1 / 2}\left\{\mathrm{E}|\varphi(\eta)|+C_{p}\left[\mathrm{E} \varphi^{2}(\eta)\right]^{1 / 2} m(p)^{1 / 2}\right\}+\Delta_{n}^{(p+1) / 2} \mathrm{E}|\varphi(\eta)|^{p+1} .
$$

Therefore,

$$
\mathrm{E}\left|\hat{P}_{n}(f)-\hat{Q}_{n}(f)\right| \leqslant C^{\prime \prime} \Delta_{n}^{1 / 2} .
$$

Thus $\left|\hat{P}_{n}(f)-\hat{Q}_{n}(f)\right|=\Delta_{n}^{1 / 2} O_{\mathbf{P}}(1)$. So, the presence of a drift function $\varphi$ in the $\left(Y_{t}\right)$ model induces a bias of order $\Delta_{n}^{1 / 2}$. We see that

$$
\hat{P}_{n}(f)-\hat{Q}_{n}(f) \underset{n \rightarrow \infty}{\rightarrow} 0 \text { in probability, }
$$

and, if $n \Delta_{n}^{2} \rightarrow 0$,

$$
\left(n \Delta_{n}\right)^{1 / 2}\left[\hat{P}_{n}(f)-\hat{Q}_{n}(f)\right] \underset{n \rightarrow \infty}{\rightarrow} 0 \text { in probability. }
$$

This achieves the proof of Theorem 4.

\section{Extension to some related models}

Usually, in the financial models, the conditional instantaneous variance of the observed diffusion $\left(Y_{t}\right)$ is given by $\sigma_{t}^{2}$ and, depending on the researchers, either $\sigma_{t}$ or $V_{t}=\sigma_{t}^{2}$ is ruled by a stochastic differential equation. The second case has been studied above. However, this framework puts aside some models (see, for example, Example 4 in Section 6). This is the reason why we study the extension to the case where the stochastic differential equation involves $\sigma_{t}$. 
Let $\left(Y_{t}, \sigma_{t}\right)_{t \geqslant 0}$ be the two-dimensional diffusion process defined by

$$
\begin{gathered}
\mathrm{d} Y_{t}=\sigma_{t} \mathrm{~d} B_{t}, \quad Y_{0}=0, \\
\mathrm{~d} \sigma_{t}=b\left(\sigma_{t}\right) \mathrm{d} t+a\left(\sigma_{t}\right) \mathrm{d} W_{t}, \quad \sigma_{0}=\xi,
\end{gathered}
$$

with $\left(B_{t}, W_{t}\right)_{t \geqslant 0}$ a standard Brownian motion of $\mathbb{R}^{2}$, and $\xi$ independent of $\left(B_{t}, W_{t}\right)_{t \geqslant 0}$. Note that $\mathrm{d} Y_{t}=\sigma_{t} \mathrm{~d} B_{t}$ and $\mathrm{d} Y_{t}=\left(\sigma_{t}^{2}\right)^{1 / 2} \mathrm{~d} B_{t}$ gives the same distribution for the process $Y_{t}$. Now we assume the following.

(A'1) Functions $b$ and $a$ are $C^{1}$ on $\mathbb{R}$ and such that

$$
\exists k>0, \quad \forall u \in \mathbb{R}, \quad b^{2}(u)+a^{2}(u) \leqslant k\left(1+u^{2}\right) .
$$

(A'2) The function $s(u)$ of diffusion $\sigma_{t}$ is defined as in (5) but now for $u$ in $\mathbb{R}$, and

$$
\int^{+\infty} s(u) \mathrm{d} u=\int_{-\infty} s(u) \mathrm{d} u=+\infty, \quad \tilde{M}=\int_{-\infty}^{+\infty} \frac{\mathrm{d} u}{a^{2}(u) s(u)}<+\infty .
$$

$\left(\mathrm{A}^{\prime} 3\right)$ Let $\tilde{\pi}(u)=1 / \tilde{M} a^{2}(u) s(u)$ denote the stationary distribution of $\sigma_{t}$ on $\mathbb{R}$. Assumption (A3) therefore becomes as follows: the initial random variable $\xi$ has distribution $\tilde{\pi}(u) \mathrm{d} u$.

(A'4) We assume that $\tilde{\pi}$ verifies

$$
\exists \alpha \geqslant 4, \quad \int_{\mathbb{R}}|u|^{\alpha} \tilde{\pi}(u) \mathrm{d} u<+\infty
$$

We consider now the class of functions $\mathscr{F}_{\alpha / 2}$ (see (10) and (11) for the definition of $\mathscr{F}_{\gamma}$, and (9) for the definition of $\left.h_{f}(v)\right)$. Denote the $p$ th moment of $\tilde{\pi}$ by

$$
\tilde{m}(p)=\int_{\mathbb{R}} u^{p} \tilde{\pi}(u) \mathrm{d} u \text { for } 0 \leqslant p \leqslant \alpha .
$$

From the observations $Y_{t_{i}}$ defined by (29), we consider the same variables $X_{i}$ as defined in (1). Now, let us denote

$$
P_{\tilde{\pi}}(f)=\int_{\mathbb{R}} h_{f}\left(u^{2}\right) \tilde{\pi}(u) \mathrm{d} u .
$$

The main difference here is that $P_{\tilde{\pi}}$ is the distribution of $\epsilon|\xi|$, where $(\epsilon, \xi)$ has distribution $\mathscr{N}(0,1) \otimes \tilde{\pi}(u) \mathrm{d} u$. Note that

$$
\mathrm{E} h_{f}\left(\xi^{2}\right)=\mathrm{E} f(\epsilon|\xi|)=P_{\tilde{\pi}}(f)
$$

Let us define

$$
\begin{gathered}
\tilde{A}_{f}(v)=\int_{-\infty}^{v}\left[h_{f}\left(u^{2}\right)-P_{\tilde{\pi}}(f)\right] \tilde{\pi}(u) \mathrm{d} u, \quad v \in \mathbb{R}, \\
\tilde{V}(f)=4 \tilde{M} \int_{\mathbb{R}} s(u) \tilde{A}_{f}^{2}(u) \mathrm{d} u .
\end{gathered}
$$

Then, the following holds. 
Theorem 5. Assume $\left(\mathrm{A}^{\prime} 1\right)-\left(\mathrm{A}^{\prime} 4\right)$ and that $f$ belongs to $\mathscr{F}_{\alpha / 2}$. Now, if $\Delta_{n} \rightarrow 0$ and $n \Delta_{n} \rightarrow+\infty$, then

(i) $\hat{P}_{n}(f) \rightarrow P_{\tilde{\pi}}(f)$ in probability, as $n$ tends to infinity and

(ii) if moreover $n \Delta_{n}^{2} \rightarrow 0$ and $\tilde{V}(f)<+\infty$,

$$
\left(n \Delta_{n}\right)^{1 / 2}\left[\hat{P}_{n}(f)-P_{\tilde{\pi}}(f)\right] \underset{n \rightarrow+\infty}{\stackrel{\mathscr{D}}{\rightarrow}} \mathscr{N}(0, \tilde{V}(f)) .
$$

If $f(x)=x^{2}$, the additional assumption $n \Delta_{n}^{2} \rightarrow 0$ is not needed.

The proof is a minor generalization of our earlier results and is omitted. As previously for Theorem 2, Theorem 5 holds whenever $\tilde{V}(f)<+\infty$. The useful sufficient conditions which allow one to check this property are now the following:

$$
\begin{aligned}
& \int_{-\infty} \mathrm{d} v s(v)\left(\int_{-\infty}^{v}|u|^{\gamma / 2} \tilde{\pi}(u) \mathrm{d} u\right)^{2}<+\infty \\
& \int^{\infty} \mathrm{d} v s(v)\left(\int_{v}^{+\infty}|u|^{\gamma / 2} \tilde{\pi}(u) \mathrm{d} u\right)^{2}<+\infty
\end{aligned}
$$

Then, if (i) and (ii) hold, for all $f \in \mathscr{F} \alpha / 2, \tilde{V}(f)<+\infty$. As in Lemma 4, these conditions are deduced from upper bounds for $\tilde{A}_{f}(v)$ near $-\infty$ and $+\infty$.

\section{Examples}

Several parametric models for the stochastic volatility $V_{t}$ have been proposed by various workers. We give below some examples which are all included in the above framework. Assumptions (A1)-(A4) are readily checked and the stationary distribution $\pi$, is, in each case, a simple and classical distribution whose moments can be easily computed.

Now, the results of the previous sections show that the moments of the distribution $P_{\pi}$ can be consistently estimated by means of empirical moments built from the observations. An important point is that the $2 p$ th moments of $P_{\pi}$ are linked to the $p$ th moments of $\pi$ by the relation

$$
\int x^{2 p} \mathrm{~d} P_{\pi}(x)=C_{2 p} \int v^{p} \pi(v) \mathrm{d} v=C_{2 p} m(p)
$$

(see (7) and (13)).

Therefore all the moments of the distribution $\pi$ can be consistently estimated. Details and simulation results will be investigated in a separate paper. Let us now consider the examples.

Example 1 (Heston 1993).

$$
\mathrm{d} V_{t}=\frac{c^{2}}{2}\left(a-\lambda V_{t}\right) \mathrm{d} t+c V_{t}^{1 / 2} \mathrm{~d} W_{t} .
$$


Assumption (A2) holds if $\lambda>0$ and $a \geqslant 1$. The stationary distribution $\pi(v) \mathrm{d} v$ of $\left(V_{t}\right)$ is the standard gamma distribution with parameters $a$ and $\lambda$ :

$$
\pi(v)=\frac{\lambda^{a}}{\Gamma(a)} \exp (-\lambda v) v^{a-1} 1_{(v>0)} .
$$

This distribution has moments of order $p$ for all positive $p$. Hence (A4) holds. These moments are given by

$$
m(p)=\frac{\Gamma(a+p)}{\Gamma(a) \lambda p} .
$$

Example 2. This example is the diffusion approximation of a discrete $\operatorname{GARCH}(1,1)$ model:

$$
\mathrm{d} V_{t}=\frac{c^{2}}{2}\left(\lambda-a V_{t}\right) \mathrm{d} t+c V_{t} \mathrm{~d} W_{t} .
$$

Assumption (A2) holds if $\lambda>0$ and $a>-1$. The stationary distribution $\pi$ of $\left(V_{t}\right)$ is the distribution with density

$$
\pi(v)=\frac{\lambda^{a+1}}{\Gamma(a+1)} v^{-a} \exp \left(-\frac{\lambda}{v}\right) \frac{1}{v^{2}} 1_{v>0} .
$$

Hence, it is the distribution of $1 / X$ where $X$ is a random variable distributed according to a gamma distribution with parameters $a+1$ and $\lambda$. The moments of $\pi$ are defined by

$$
\begin{gathered}
m(p)=\lambda^{p} \frac{\Gamma(a+1-p)}{\Gamma(a+1)} \text { if } \quad p<a+1, \\
m(p)=+\infty \quad \text { if } \quad p \geqslant a+1 .
\end{gathered}
$$

\section{Example 3. (Chesney and Scott 1989).}

$$
V_{t}=\exp \left(X_{t}\right), \quad \mathrm{d} X_{t}=\alpha\left(\beta-X_{t}\right) \mathrm{d} t+\rho \mathrm{d} W_{t} .
$$

Clearly, since $\left(X_{t}\right)$ is an Ornstein-Uhlenbeck diffusion process with stationary distribution $\mathscr{N}\left(\beta, \rho^{2} / 2 \alpha\right)$, if $\alpha>0,\left(V_{t}\right)$ is ergodic with a log-normal stationary distribution which has moments of any order, given by

$$
m(p)=\exp \left(p \beta+\frac{p^{2}}{2} \frac{\rho^{2}}{2 \alpha}\right) .
$$

\section{Example 4 (Scott 1987; Stein and Stein 1991).}

$$
V_{t}=\sigma_{t}^{2}, \quad \mathrm{~d} \sigma_{t}=\alpha\left(\beta-\sigma_{t}\right) \mathrm{d} t+\rho \mathrm{d} W_{t} .
$$

Here, when $\alpha>0,\left(\sigma_{t}\right)$ is an ergodic diffusion on $\mathbb{R}$ (see Section 5$), \tilde{\pi}(u)$ is the distribution $\mathscr{N}\left(\beta, \rho^{2} / 2 \alpha\right)$ and 


$$
\tilde{m}(2 p)=\mathrm{E}\left[\beta+\left(\frac{\rho^{2}}{2 \alpha}\right)^{1 / 2} \epsilon\right]^{2 p},
$$

where $\epsilon$ has distribution $\mathscr{N}(0,1)$. When $\beta=0$, this is a special case of Example 1 .

\section{References}

Bibby, B. and Sorensen, M. (1995) Martingale estimation functions for discretely observed diffusion processes. Bernoulli, 1, 17-39.

Black, F. and Scholes, M. (1973) The pricing of options and corporate liabilities. J. Political Economy, 81, 637-659.

Bollerslev, T., Chou, R.Y. and Kroner, K.F. (1992) Arch modeling in finance. A review of the theory and empirical evidence. J Econometrics, 52, 5-59.

Chesney, M. and Scott, L. (1989) Pricing European currency options: a comparison of the modified Black-Scholes model and a random variance model. J. Financial Quantitative Anal., 24, 267289.

Dacunha-Castelle, D. and Florens-Zmirou, D. (1986) Estimation of the coefficient of a diffusion from discrete observations. Stochastics, 19, 263-284.

Donahl, G. (1987) On estimating the diffusion coefficient. J. Appl. Probab., 24, 105-114.

Elliott, R.J., Lakhdar, A. and Moore, J.B. (1995) Hidden Markov models: estimation and control. Applic. Math. Springer.

Florens-Zmirou, D. (1984) Théorème de limite centrale pour une diffusion et sa discrétisée. C. $R$. Acad. Sci. Paris, Sér. I, 299, 995-998.

Foster, D.P. and Nelson, D.B. (1994) Asymptotic filtering theory for univariate ARCH models. Econometrica, 62, 1-41.

Genon-Catalot, V. (1990) Maximum contrast estimation for diffusion processes from discrete observations. Statistics, 21, 99-116.

Genon-Catalot, V. and Jacod, J. (1993) On the estimation of the diffusion coefficient for multidimensional diffusion processes. Ann. Inst. Henri Poincaré, Probab. Statist., 29, 119-151.

Heston, S.L. (1993) A closed-form solution for options with stochastic volatility with applications to bond and currency options. Rev. Financial Studies, 6, 327-343.

Hull, J. and White, A. (1987) The pricing of options on assets with stochastic volatilities. J. Finance, 42, 281-300.

Kessler, M. (1995) Estimation of an ergodic diffusion from discrete observations. Prépublication 306, Laboratoire de Probabilités, 1'Université Paris VI.

Kutoyants, Yu.A. (1984) Parameter Estimation for Stochastic Processes. Berlin: Heldermann.

Larédo, C. (1990) A sufficient condition for asymptotic sufficiency of incomplete observations of a diffusion process. Ann. Statist., 18, 1158-1171.

Leblanc, B. (1996) Une approche unifiée pour une forme exacte du prix d'une option dans les différents modèles à volatilité stochastique. Stochastics Stochastics Rep., 57, 1-35.

Nelson, D.B. (1992) Filtering and forecasting with misspecified ARCH models I (Getting the right variance with the wrong model). J. Econometrics, 52, 61-90.

Rogers, L.C.G. and Williams, D. (1987) Diffusions, Markov Processes, and Martingales, Vol. 2, Ito Calculus. Wiley Series in Probability and Mathematical Statistics. New York: Wiley.

Scott, L.O. (1987) Option pricing when the variance changes randomly: theory, estimation and an application. J. Financial Quantitative Anal., 22, 419-438. 
Stein, E.M. and Stein, J.C. (1991) Stock prices distributions with stochastic volatility: an analytic approach. Rev. Financial Studies, 4, 727-752.

Taylor, S.J. and Xu, X. (1994) The magnitude of implied volatility smiles: theory and empirical evidence for exchange rates. Rev. Futures Markets, 13, 355-380.

Taylor, S.J. and Xu, X. (1995) The incremental volatility information in one million foreign exchange quotations. In Preprints of Conference on High Frequency Data in Finance, Zürich, 1995.

Wiggins, J.B. (1987) Option values under stochastic volatilities. Theory and empirical evidence. J. Financial Economics, 19, 351-372.

Received March 1996 and revised March 1997 